\title{
Contextual Ontology: A Storage Tool for Extracting Context from Web Pages
}

\author{
Nidhi Tyagi \\ Shobhit University, \\ Meerut,India.
}

\author{
Rahul Rishi \\ Maharishi Dayanand \\ University, \\ Rohtak, India.
}

\author{
R.P. Agarwal \\ Shobhit University, \\ Meerut, India.
}

\begin{abstract}
Contextual ontology helps in the knowledge-full indexing of documents, providing semantic structure to the document. The named entity and the associated keywords can be a clue to the contextual sense of the documents in the corpus. The proposed technique for the information retrieval creates the ecology of ontology which stores the ontological representation of the crawled documents. Further, the efficiency of ontology based indexing is enhanced as compared to the traditional key-word based retrieval system.
\end{abstract}

General terms: context, domain, retrieval, information, storage.

Keywords: Contextual, Ontology, indexer, hyponyms.

\section{INTRODUCTION}

The information on the web is available in various formats, such as RDF, XML and HTML. Some of the representations provide semantic knowledge while others only indicates the structure and lay-out of documents [1]. Context based retrieval system has received a great deal of attention in recent years. With the help of context ontology the relationship among the concepts in a text and its knowledge in various applications can be used. The practice of the context can minimize ambiguity in searching and provides foundation to effective information retrieval system. The idea of context can be applied at the user-interface end as well as to the documents which are in the repository, and the index created on this scheme can provide improved precision and recall value of crawled web pages. The traditional keyword based indexing of the documents is time consuming and may provide indistinguishable and redundant information to the user. Documents stored in the repository without the context may lead to futile results causing large memory utilization which reduces the precision and recall values.

\subsection{0ntology}

Ontology is defined as precise specification of a shared conceptualization. A domain ontology (or domain-specific ontology) models a specific domain, which represents part of the real world. Specific meanings of term applied to domain are provided by domain ontology as the word 'spider' which has many different interpretations. Ontology about the domain of human character; would deduce the "spider-man" meaning of the word, while ontology about the domain of animal would deduce the "spider-monkey" and on the other hand ontology of the domain of computer may refer to the "computer program".

The domain ontology can be further extended by providing the context ontology to represent and understand the real world entity in the more precise and clear way. Such ontology provides a vocabulary for representing knowledge about a domain and for describing specific situations in the domain as it shares the context information in a pervasive computing domain and include machine-understandable definitions of basic concepts in the domain and relations among them. The advantage of such model is sharing common understanding of the structure of context information among users, devices and services to enable semantic interoperability. It also enables reuse of domain knowledge, i.e., building a large ontology by integrating several ontologies describing portions of the larger domain. Secondly, it enables formal analysis of domain knowledge, for example, context reasoning becomes possible by clearly defining context ontology.

\section{RELATED WORK}

The literature survey reveals that, there have been few significant attempts to merge information retrieval and ontological models, [2] proposed a text processing system to build ontological domain. Machine learning techniques proposed in [3] have been widely used for automatically extracting semantic information and also for text categorization. Context models have been classified into informal and formal models. Informal context models are often based on proprietary representation schemes which have no facilities to ease shared understanding about context between different systems. The systems with informal context models, as discussed in [4], represents context in form of attribute-value tuples, where as, [5] proposed a Web based model of context in which each object has a corresponding Web description. Formal context models commonly employ formal modeling approaches to manipulate context. The research paper [6] model context using both ER and UML models; context can be easily managed with relational databases and [7] represented context in Gaia system as firstorder predicates written in DAML+OIL. Existing formal context models support formality and address a certain level of context reasoning. However, none of them has addressed formal knowledge sharing, or has shown a quantitative evaluation for the feasibility of context reasoning in pervasive computing environments, where we always have to face resource-constraint devices. Yet another research paper, [8] present an ontology-based formal context model to address critical issues including formal context representation, knowledge sharing and logic based context reasoning. Through performance analysis, it reveals quantitative evaluation for context reasoning in pervasive computing environments. The research paper [1] proposed the technique for providing semantic structure to the HTML documents and stores it in the knowledge base as predicates, which helps in the retrieval of context related documents.

The critical look at the available literature reveals that there is a requirement of such a system which generates the contextual ontology for the different hyponyms, so that the words having the multiple interpretations can be represented in a more 
meaningful and logical manner to remove the ambiguity for representation both for the search- engine as well as the user.

\section{PROPOSED WORK}

The need to access diverse data sources becomes especially acute when undertaking synthetic analyses to address exact topic specific questions. This requires accuracy and precise technique in organizing the web documents and answering the queries. In the proposed method, the submitted query after being preprocessed is mapped to the indexer, which matches to various ontologies existing in the ecology of ontology. The ecology of ontology acts as a knowledge base, created from the well organized web documents, used by the indexer to index the results to the user. The ecology of ontologies is maintained at various levels as domain level and then contextual level as it moves down the hierarchy. The domain for the search made by the user is identified by the set of key words submitted. The documents are organized using the bottom up approach, by identifying the keywords, followed by the context formation and finally the domain identification as shown in figure 3 . The representation can be done through the semantic net or frames. The concept of context can be applied at the user-interface end as well as at maintaining the documents in the repository, on the bases of which indexing can be provided for improving the precision and recall value of crawled web pages. The traditional keyword base indexing of the documents is more time consuming and may provide vague and irrelevant information to the user. User's queries without the context may lead to fruitless results. The proposed method generates the contextual ontology for the different hyponyms. The figure 1 represents the architecture for extracting accurate documents as per the requirements of the user.

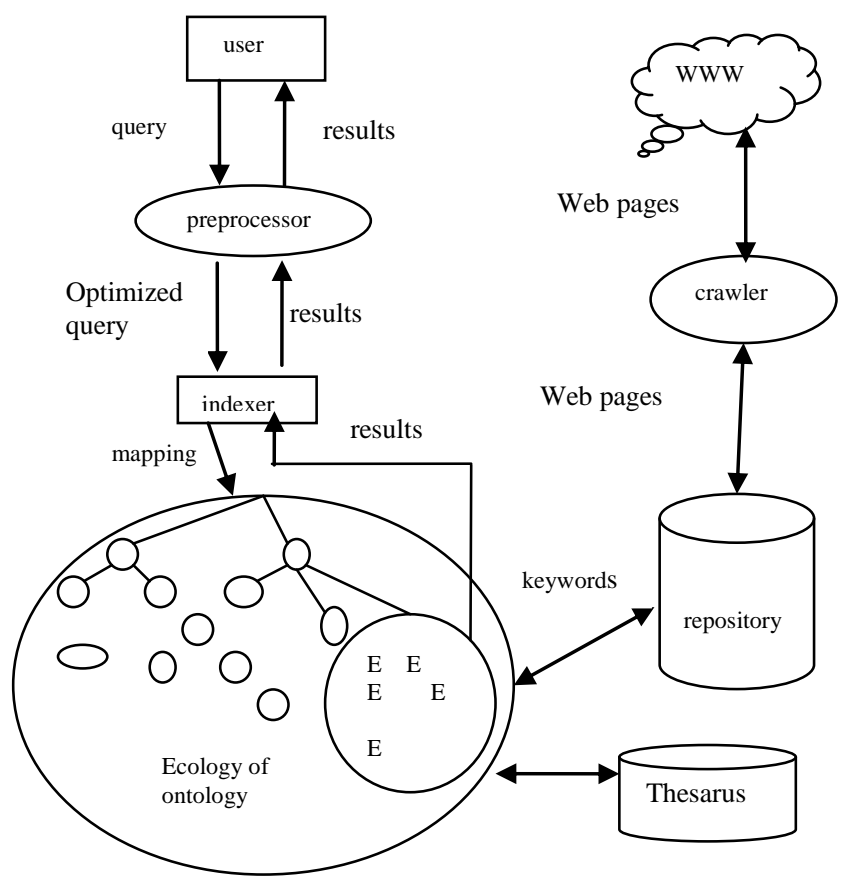

Figure 1: Architecture Ontology based contextual information retrieval.

\subsection{Description of the various components}

1. Ecology of ontology: This module provides an environment of various ontologies, where semantic representation of all the ontologies generated from the retrieved documents is represented. The approach for the ontologies is bottom-up, from the set of keywords the context is identified and further the domain of the document. In this module the ontologies are represented in the form of frames, for the various hyponyms. Category, characteristics, events and synonyms are identified for such words and finally the context is rectified.

2. Indexer: This module is important as it provides the ontology based index for the retrieval, which is different from others since it takes care of context and meaning of term. Data structured like hash table, binary tree AVL tree [9] etc. can be used to represent the indexer. It provides faster access to the user's request. It follows the top to bottom approach by first identifying the domain and further the context of the related query.

3. Pre-processor: The preprocessing step involves stemming as well as removal of stop words. A stop word is any word which has no semantic content. Common stop words are prepositions and articles, as well as high frequency words that do not help retrieval.

4. Thesaurus: The dictionary of words available on the World Wide Web from thesaurus.com which contains the words as well as their multiple meanings.

5. Repository. This is a database which contains the various contexts. Also the new contexts derived from thesaurus are stored in this repository. The context repository maintains a database of several types of context data.

\subsection{Algorithm}

Begin

Read a URL from the set of seed URLs

Download the document

Check whether the document has

Already been downloaded or not,

If the document is a fresh one

Then

Save it in repository

Begin

Preprocess the document,

Identify the keywords

Identify the hyponyms

Generate the contextual ontology

Deduce the domain of the document.

Maintain ecology of ontology. 
End.

Else

\section{Discard}

End.

Figure 2: Algorithm for contextual ontology.

The extracted information from the document downloaded by the crawler is fragmented on the bases of the various criteria, which assist in determining the context and finally the domain of the document. Storing the documents on the bases of the context provides more efficient results. Figure 3 shows how the document is analyzed to extract the vital information that is relevant to provide the semantic representation to the document.

Document

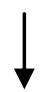

Set of Keywords

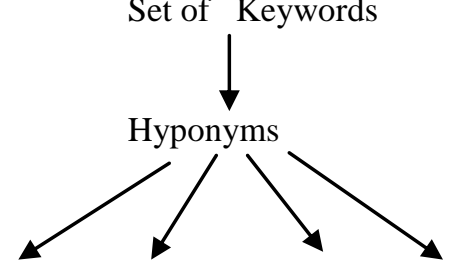

Category characteristics events synonyms

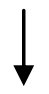

Domain of document

Figure 3: Structure of document ontology

\subsection{Example}

Suppose the keyword is "spider". On searching the documents related to this keyword, number of related information is available, as shown in figure 4.

The specified keyword is a hyponyms i.e. multiple contextual sense, as : (i) Insect (ii) Crawler (iii) Game

(iv) Monkey (v) Fiction.

The web pages that are crawled from www if arranged on the bases of the hyponyms, will give more meaningful representation to the documents. The details of the document retrieved (D1, D2, D3) are analyzed and represented in form of frames to give the semantic representation to documents, as shown in the figure 5 .

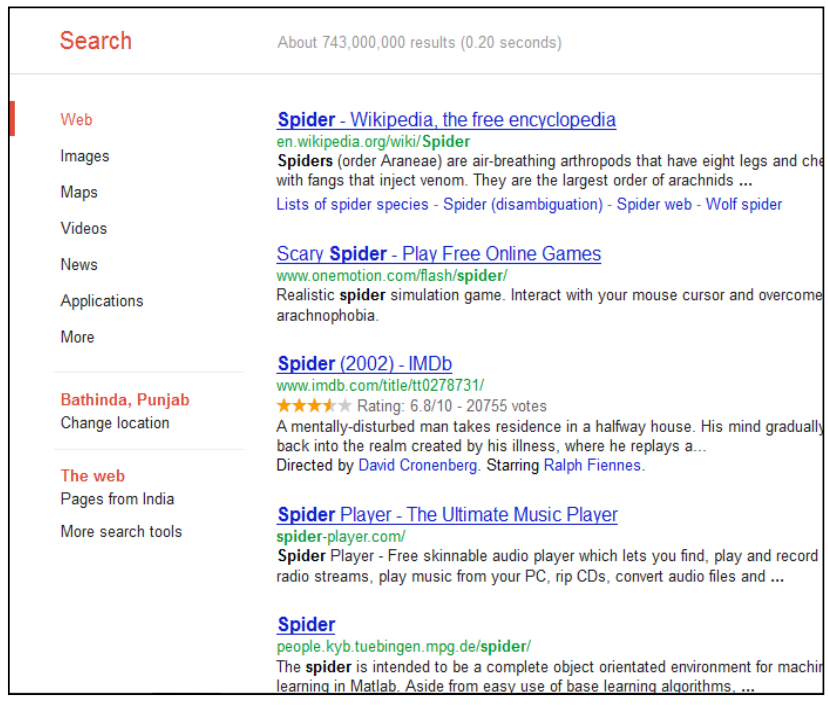

Figure 4: Documents retrieved for keyword "Spider"

\begin{tabular}{|l|}
\hline Keywords: Spider, segment, web, pesticide, body \\
\hline Hyponyms: Spider \\
\hline Category: Insect \\
\hline $\begin{array}{l}\text { Characteristics : arthropods, eight-legs, body- } \\
\text { segmented, no antennae }\end{array}$ \\
\hline Events: 40.000 species. 109 families. \\
\hline Synonyms : bug, pest, creepy-crawly, creature \\
\hline
\end{tabular}

\begin{tabular}{|l|}
\hline Keywords: Crawler, spider, download, search s/w \\
\hline Hyponyms: Spider \\
\hline Category: computer program \\
\hline Characteristics :, browse www, harvest e-mail \\
\hline Events: Mercator, incremental, hidden-web \\
\hline Synonyms : worm, crawler, ants \\
\hline
\end{tabular}

\begin{tabular}{|l|}
\hline Keywords: spider, video, game, developer, play- \\
\hline Hyponyms: Spider \\
\hline Category: on-line game \\
\hline Characteristics : online, released on Xbox 360, \\
\hline Events: spider_man $1 . . . .$. \\
\hline Synonyms : sport, amusement \\
\hline
\end{tabular}

Figure 5: Semantic representation for "spider" using Frames

The ontology is constructed showing the relationships and the associated words in different context, with the help of the frames as in figure 6 . 


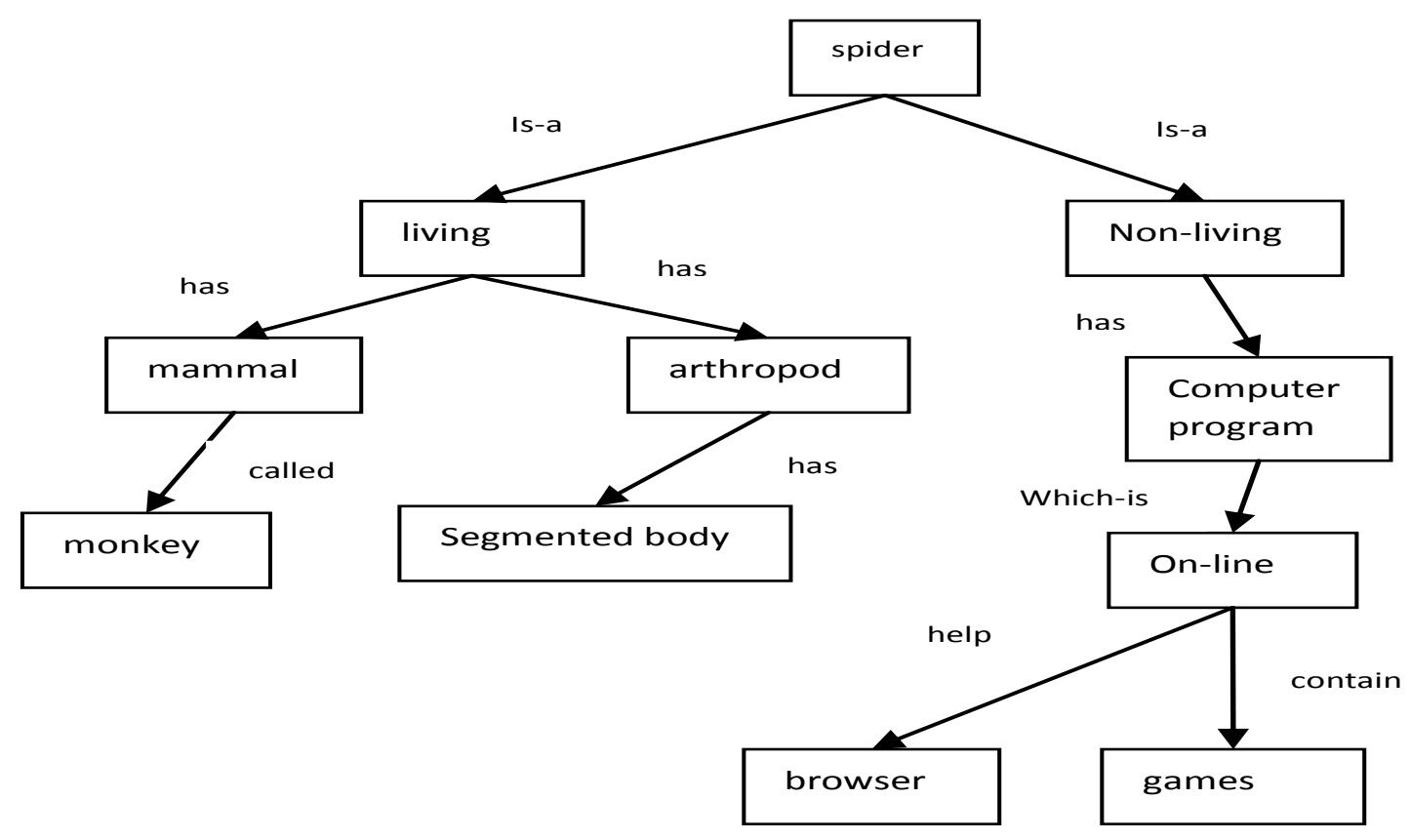

Figure 6: Ontological format of keyword "Spider".

On retrieving the information related to the keyword "spider" documents having different context are collected. But to be more precise if the query submitted by the user is transformed to semantic or predicate form as proposed in [1] the reply will be more accurate.

Based on the above technique, various documents in reference to different contextual sense were crawled .The table below shows the no. of documents available for different context of the keyword "spider".

$\begin{array}{lr}\text { keyword } & \text { no. of documents } \\ \text { Spider } & 439000000 \\ \text { Spider insect } & 13000000 \\ \text { Spider man } & 252000000 \\ \text { Spider monkey } & 5450000 \\ \text { Spider game } & 278000000\end{array}$

The histogram drawn for the above available data is represented in figure 7 , and it is quite evident that if the documents are managed in the repository ontologically and if the user's query is also in predicate form the resulting relevant documents are much lesser and the availability time will also decrease, while on the other side traditional keyword search is The traditional keyword base search of the documents is more time consuming and may provide indistinguishable and redundant information to the user.

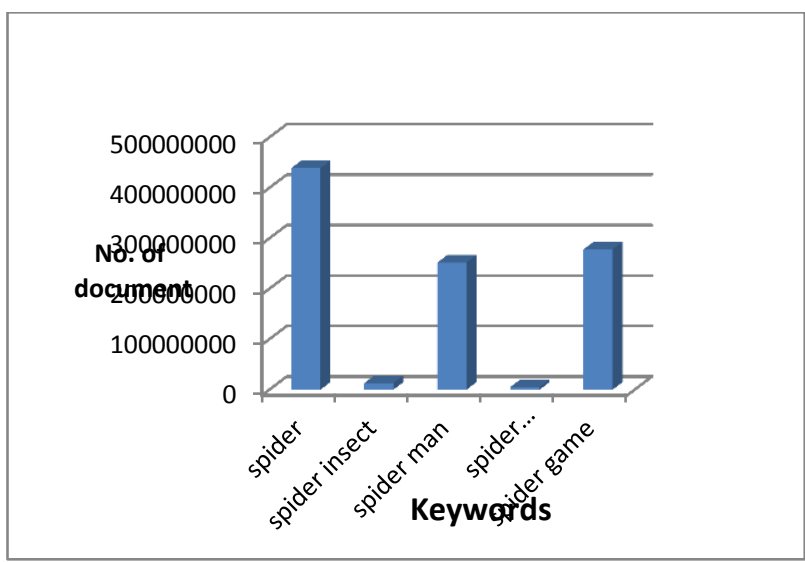

Figure 7: Histogram showing the comparison between contextual searches for the keyword "spider".

\section{CONCLUSION}

The proposed method for the information retrieval creates the ecology of ontology which stores the ontological representation of the crawled documents which acts as a knowledge base used by the indexer to organize the documents. The ontologies are maintained at various levels as domain level and then contextual level as it moves down the hierarchy. If the user's query is also in predicate form the resulting relevant documents will be much lesser and the availability time required will also decrease. Thus, ontology based indexing enhances the efficiency and than traditional key-word based retrieval system. 


\section{REFERENCES}

[1] Nidhi Tyagi , Rahul Rishi and R.P. Agarwal,"Semantic Structure Representation of HTML Document Suitable for Semantic Document Retrieval", International Journal of Computer Applications (0975 - 8887) Volume 46No.13, May 2012.

[2] L. Weihua, "Ontology supported intelligent information agent", proceeding IEEE Symp, on Intelligent Systems, pages383-387, IEEE,2002.

[3] LIOA Shou-yi and DAI Jin-hai, "Design and implementation of communication system in agent-based distributed simulation ", Simulation Journal of system, 18(4), 2006.

[4] A.K.Dey, et al. "A Conceptual Framework and a Toolkit for Supporting the Rapid Prototyping of Context- Aware Applications", Human- computer Interaction Journal, Vol. 16(2-4), pp. 97-166, 2001.

[5] Tim Kindberg, et al. "People, Places, Things: Web Presence for The Real World", Technical Report HPL2000-16, HP Labs, 2000.
[6] Karen Henricksen, et al."Modeling Context Information in Pervasive Computing Systems", Pervasive 2002.

[7] Anand Ranganathan, et al. "A Middleware for ContextAware Agents in Ubiquitous Computing Environments",USENIX International Middleware Conference, 2002.

[8] Xiao Hang Wang, Da Qing Zhang, Tao Gu1,Hung Keng Pung "Ontology Based Context Modeling and Reasoning using OWL", Second IEEE Annual Conference on Pervasive Computing and Communications Workshops, 2004

[9] Nidhi Tyagi, Rahul Rishi and R.P.Agrawal," Context based Web Indexing for Storage of Relevant Web Pages", International Journal of Computer Applications (0975 - 8887) Volume 40- No.3, February 2012.

[10] Parul Gupta and A.K.Sharma," Context based Indexing in Search Engines using Ontology", International Journal of Computer Applications,Volume 1 No. 14, pp 49-52, 2010. 